\title{
Retrospective review of maternal deaths and maternal near misses due to major obstetric haemorrhage at a tertiary care centre in India
}

\author{
Swati Agrawal, Anuradha Singh*, Ratna Biswas, Abha Singh
}

Department of Obstetrics and Gynaecology Lady Hardinge Medical College and SSK Hospital, New Delhi, India

Received: 10 March 2019

Accepted: 01 July 2019

\section{*Correspondence:}

Dr. Anuradha Singh,

E-mail: sbxy@rediffmail.com

Copyright: (C) the author(s), publisher and licensee Medip Academy. This is an open-access article distributed under the terms of the Creative Commons Attribution Non-Commercial License, which permits unrestricted non-commercial use, distribution, and reproduction in any medium, provided the original work is properly cited.

\begin{abstract}
Background: Maternal near miss (MNM) is now widely accepted as a better indicator of maternal health than maternal death and reflects the quality of obstetric care in a particular institution.

Methods: This is a retrospective study conducted at Lady Hardinge Medical College and Smt. Sucheta Kriplani Hospital over a period of 12 months (April 2016-March 2017), of all cases of maternal death and near miss maternal deaths due to major obstetric haemorrhage $(\mathrm{MOH})$.

Results: During the period reviewed, there were 13,083 deliveries, 12,958 live births and 37 maternal deaths. There were 30 cases of near miss maternal deaths and 2 maternal mortalities due to $\mathrm{MOH}$. The mortality index was $6.25 \%$. Severe maternal outcome ratio (SMOR) was 2.46 .

Among the near miss cases $(n=30)$, morbidly adherent placenta was the cause in $26.6 \%$ of cases(n=8), postpartum hemorrhage in $23 \%$ of cases( $(n=7)$; rupture uterus in $13 \%$ cases $(n=4)$; massive abruption in $13 \%$ of cases(n=4) and placenta praevia with antepartum haemorrhage in $3 \%$ of cases $(n=1)$. Early obstetric haemorrhage due to ruptured ectopic pregnancy and incomplete abortion resulted in MNM in 16\% $(n=5)$ and $3.3 \%(n=1)$ cases respectively.

It was observed that in $40 \%(n=12)$ of MNM cases ( 8 cases of morbidly adherent placenta plus 4 cases of rupture uterus), previous cesarean section was the single most important causative factor for the morbidity of the patient.

Conclusions: Reduction in cesarean section rates is imperative to reduce morbidity and mortality associated with $\mathrm{MOH}$.
\end{abstract}

Keywords: Cesarean section, Major obstetric haemorrhage, Maternal near miss, Mortality index

\section{INTRODUCTION}

Maternal Death Surveillance \& Response (MDSR) is a continuous cycle of identification, notification and review of maternal deaths followed by actions to improve quality of care and prevent future deaths. Maternal near miss (MNM) is defined by survival of a life threatening complication during pregnancy, delivery, or within 42 days of termination of pregnancy. Over the recent years, maternal near miss (MNM) has emerged as a better indicator of maternal health than maternal death for various reasons: Near miss cases are much more frequent than maternal deaths and reflect the quality of obstetric care in a particular institution. Further, the causative factors are the same for both MNM and maternal deaths (MD), so a review of MNM can identify the gaps of health infrastructure and aid in improving the quality of healthcare.

India's current Maternal Mortality Ratio (MMR) stands at 167 deaths per 100,000 live births. The government of India (GOI) aims to reduce the MMR to 100 by the year 2020. To achieve this aim, GOI is strengthening its MDSR program and MNM review by introducing improved MDSR guidelines which emphasize on no 
name, no blame policy; introduction of confidential reviews and review of migrant maternal deaths.

Major Obstetric haemorrhage $(\mathrm{MOH})$ is defined as blood loss more than 2 liters or rate of blood loss of more than $150 \mathrm{ml} / \mathrm{min}$ or $50 \%$ blood volume loss within 3 hours. The global incidence of $\mathrm{MOH}$ is said to $3.7-5 / 1000$ births. ${ }^{1}$ The incidence of $\mathrm{MOH}$ in developed countries varies widely from $0.16-8.8 \% .^{2}$ An Indian study conducted in 2015 reported the incidence of $\mathrm{MOH}$ to be 5.7/1000 births. ${ }^{3}$ According to WHO, nearly 5 women die every hour in India from complications developed during childbirth , with heavy blood loss caused by haemorrhage being a major factor.

$\mathrm{MOH}$ is one of the topmost causes of maternal mortality and maternal near misses worldwide. It is estimated that postpartum haemorrhage alone accounts for 127,000 deaths annually around the world. ${ }^{4}$

The present study is a retrospective analysis of the MD \& MNMs caused due to MOH. The authors aim to analyse the factors responsible for such life threatening $\mathrm{MOH}$ so that corrective \& preventive measures may be instituted to reduce the occurrence of such mishaps in future.

\section{METHODS}

This is a retrospective compilation of data from Lady Hardinge Medical College \& Smt. Sucheta Kriplani Hospital over a period of 12 months (April 2016-March 2017), of all cases of maternal deaths and maternal near misses occurring due to massive obstetric haemorrhage.

Data was collected from case records of patients from Medical Records Department with prior permission. The WHO document "The WHO near-miss approach for maternal health" was taken as the standard reference for reviewing the case records.

A maternal near miss is defined by survival of a lifethreatening complication during pregnancy, delivery, or within 42 days of termination of pregnancy (excluding accidental or incidental causes). Maternal mortality is defined as death during pregnancy or within 42 days after termination of pregnancy due to any cause aggravated by the pregnancy or its management (but not incidental or accidental causes).

Maternal deaths and near misses together constitute severe maternal outcomes (SMOs). The mortality index (expressed as a percentage) is the number of maternal deaths divided by the number of women with SMO. ${ }^{5}$ More the number of maternal deaths, higher is the mortality index. The maternal near miss ratio is the number of near misses per 1000 live births and the SMO ratio is the number of women with SMO per 1000 live births. ${ }^{5}$

\section{RESULTS}

During the 1 year period reviewed, there were 13,083 deliveries, 12,958 live births and 37 maternal deaths. The Maternal Mortality Ratio was 285.53/100,000 live births. There were 30 cases of maternal near miss and 1 maternal mortality due to massive obstetric haemorrhage. The mortality index i.e. the number of maternal deaths occurring amongst all women with massive obstetric haemorrhage was $3.33 \%$. Severe maternal outcome ratio (SMOR) for massive obstetric haemorrhage i.e. number of women with massive obstetric haemorrhage (MD plus MNM) per 1000 live births was 2.39 .

The mean age of the study population was 27.4 years. Majority $(54 \%)$ of the patients were multigravidae with para 2 or more. Excluding 6 cases of early pregnancy $\mathrm{MOH}$, the pregnancy was supervised in $58 \%$ of cases and $42 \%$ of patients did not have even a single antenatal visit. The average duration of hospital stay in MNM cases was 14.6 days. The mode of delivery was vaginal in $21 \%$ of cases and caesarean in $79 \%$ of cases (excluding 6 cases of early pregnancy $\mathrm{MOH}$ ). Hysterectomy was needed in 12 patients and internal iliac artery ligation was done in 10 patients.

Among the near miss cases $(n=30)$, morbidly adherent placenta was the most common cause of massive obstetric haemorrhage occurring in $26.6 \%$ of cases $(n=8)$. Other causes were postpartum hemorrhage occurring in $23 \%$ of cases $(n=7)$; rupture uterus occurring in $13 \%$ cases $(n=4)$; antepartum haemorrhage occurring due to massive abruption in $13 \%$ of $\operatorname{cases}(n=4)$ and due to placenta praevia in $3 \%$ of cases $(n=1)$. Early obstetric haemorrhage due to ruptured ectopic pregnancy and incomplete abortion resulted in MNM in $16 \%(n=5)$ and $3.3 \%(n=1)$ cases respectively (Figure1).

Analysis of cases of MNM occurring due to rupture uterus $(n=4 ; 13.3 \%)$ showed that all cases occurred in patients with previous caesarean section. The patients were in spontaneous labour. Two out of 4 patients presented to the hospital with rupture uterus while the other two ruptured during hospital stay.

Previous caesarean section was also the most important risk factor for morbidly adherent placenta and was present in all the MNM cases due to morbidly adherent placenta.

Surveillance of MNM occurring due to post-partum haemorrhage $(\mathrm{PPH})(\mathrm{n}=4 ; 13.3 \%)$ revealed that all cases of massive PPH occurred during caesarean section. The indication for doing a caesarean section was fetal distress in 3 out of 4 cases. However, it is noteworthy that 2 out of 3 babies with fetal distress had a normal APGAR score.

The above findings emphasize that caesarean section is the single most important risk factor for major obstetric 
haemorrhage leading to maternal near miss. There is an urgent need to check the alarmingly high rates of caesarean section and to audit the caesarean sections by institutions.

Patients who became MNM due to abruptio placentae $(n=4 ; 13.3 \%)$ were all multigravidae with poorly spaced pregnancies. Three out of 4 were severely anaemic at the time of presentation and presented in shock. These patients were unbooked with no antenatal supervision and it is possible that they were anaemic already and decompensated rapidly with antepartum haemorrhage.

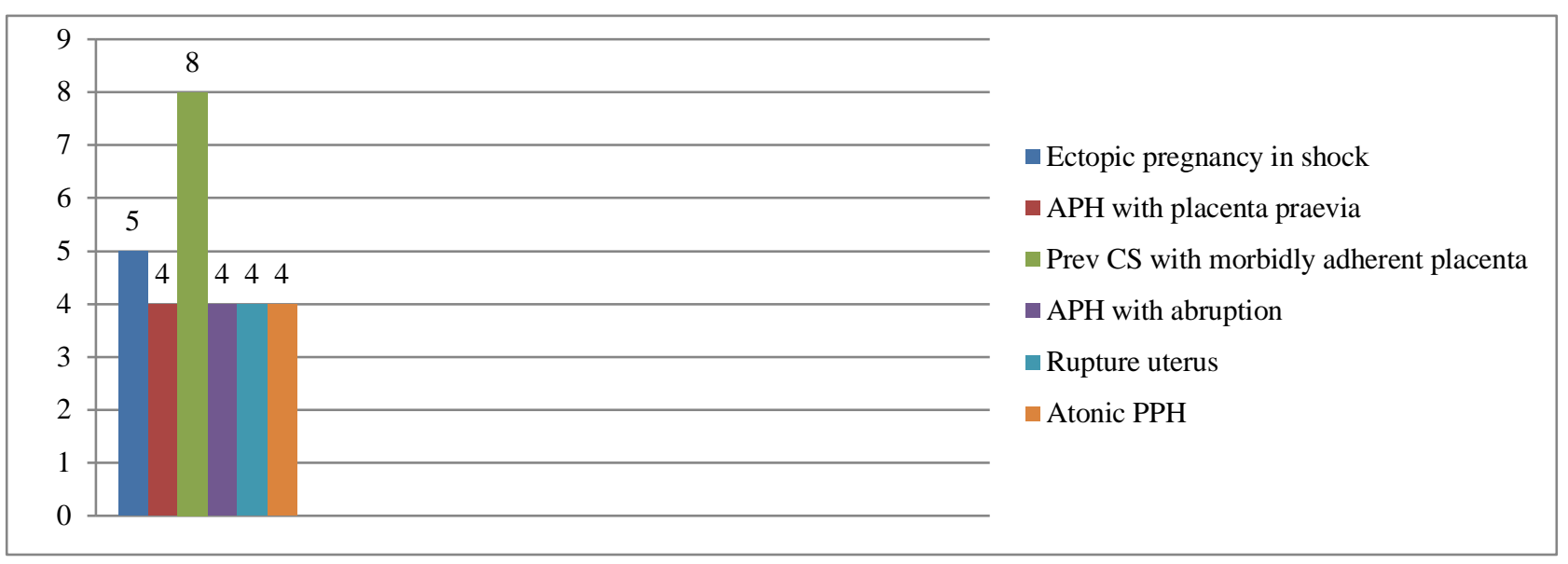

Figure 1: Causes of MOH leading to maternal near miss.

Surveillance of MNM occurring due to early pregnancy haemorrhage (ectopic pregnancy and incomplete abortion) $(\mathrm{n}=5 ; 16.6 \%)$ revealed that all patients except 1 were multigravidae (G4 or more) with poorly spaced unplanned pregnancies.

The above findings bring to light the urgency to address the unmet need for contraception so that accidental pregnancies can be avoided and the women are well nourished before becoming pregnant. Also, there is a need to strengthen the antenatal services at the grass root level so that no pregnancy ends in a complication due to lack of supervision.

The lone case of maternal death occurring due to $\mathrm{MOH}$ was a 23 year old G2P1L1 with 29 weeks twin pregnancy with dengue hemorrhagic fever with severe thrombocytopenia who presented with abruption in active labour. The patient was referred from a tertiary centre in view of non-availability of platelets and precious time was lost in transportation of the patient. The patient had massive PPH following delivery and went into irreversible shock as the replacement of blood and blood components could not be completed promptly due to the non-availability of group specific blood and blood components. The above case highlights the need to strengthen blood bank facilities at all levels so that precious time is not lost in transporting the patient for the want of blood and blood products. Also there should be regular conduct of $\mathrm{PPH}$ drills in all institutions so that the residents and all the staff members are adequately trained in managing such complications.
Since there were 2 stillbirths, and one baby died in immediate neonatal period, analysis for neonatal nursery admission is done in 107 babies. There was a total of 39 babies of 107 (36.4\%) who required nursery admission for observation either due to respiratory complications, metabolic complications or sepsis. The abnormal waveforms of umbilical artery and middle cerebral artery were also compared with neonatal nursery admission (Figure 1).

\section{DISCUSSION}

Major Obstetric Haemorrhage(MOH) is the leading cause of maternal mortality and morbidity worldwide. Several publications have documented an increase in the incidence of obstetric haemorrhage particularly postpartum hemorrhage over the recent years. ${ }^{6,7}$ It is important to identify the causative factors for $\mathrm{MOH}$ so that remedial measures can be taken to reduce their occurrence or severity.

The present study showed that the majority (54\%) of patients with severe $\mathrm{MOH}$ were multigravidae with para 2 or more and the pregnancies were unplanned. This highlights the unmet need for contraception in India. Despite a robust Family Welfare program in place, the Government of India has had limited success in keeping a check on unwanted pregnancies. A recent study conducted in 2016 revealed that there is considerable disparity in the contraceptive use amongst different states in India and more focus is needed in the states faring poorly. ${ }^{8}$ 
The present study also revealed that a significant number of women did not receive any antenatal care during pregnancy ( $42 \%$ excluding the early pregnancy cases). Lack of antenatal care is associated with a significant increase in maternal as well as neonatal morbidity and mortality. Many studies in the past have evaluated the causes for lack of prenatal care and most suggest that socioeconomic factors, educational status, access to healthcare facility and quality of healthcare are independent risk factors. ${ }^{9}$ Improving awareness regarding the importance of family welfare services and strengthening the quality of services is the only way forward.

Caesarean section was found to be a significant risk factor for severe $\mathrm{MOH}$ in the present study. It was observed that in $40 \%(n=12)$ of MNM cases ( 8 cases of morbidly adherent placenta plus 4 cases of rupture uterus), previous cesarean section was the single most important causative factor for the morbidity of the patient. Also, it was noted that all the cases of MNM due to postpartum haemorrhage $(n=7 ; 23 \%)$ occurred intraoperatively during cesarean section.

The incidence of caesarean section rates has seen a surge over the last two decades across the globe. According to a recent retrospective analysis, the caesarean section rate in India has increased from $2.9 \%$ of deliveries $1992-93$ to a rate of $17.2 \%$ in 2015-26 (according to NFHS 4 survey). ${ }^{10}$

It is obvious from the above figures that reducing cesarean section rates is the need of the hour. It is imperative now that all institutions introduce cesarean sections audit which will create awareness and initiate discussion among obstetricians regarding indications for cesarean sections and lack of necessity.

\section{Funding: No funding sources}

Conflict of interest: None declared

Ethical approval: Not required

\section{REFERENCES}

1. Chandrasekaran E, Arulkumaran S. Obstetric and intrapartum emergencies: a practical guide to management., Cambridge university. Chapter 5-
Algorithms for Management of Top Five Direct Killers-Massive Obstetric Haemorrhage; 2012:33.

2. Zhang WH, Alexander S, Bouvier-colle $\mathrm{MH}$, Macfarlane A. Incidence of severe preeclampsia, postpartum haemorrhage and sepsis as a surrogate marker for severe maternal morbidity in a European population based study: the MOMS-B survey. BJOG. 2015;112:89-96.

3. Padmasekar A, Jothy S. Retrospective study of massive obstetric haemorrhage and its materno fetal outcomes in a tertiary care centre. Int $\mathrm{J}$ Reprod Contracept Obstet Gynecol. 2017;6(2):555-7.

4. Kumar N. Postpartum hemorrhage; a major killer of woman: review of current scenario. Obstet Gynecol Int J. 2016;4(4):00116.

5. Say L, Souza JP, Pattinson RC. Maternal near misstowards a standard tool for monitoring quality of maternal health care. Best Pract Res Clin Obstet Gynaecol. 2009;23(3):287-96.

6. Lutomski JE, Byrne BM, Devane D, Greene RA. Increasing trends in atonic postpartum haemorrhage in Ireland: an 11-year population-based cohort study. BJOG. 2012;119(3):306-14.

7. Knight M, Callaghan WM, Berg C, Alexander S, Bouvier-Colle MH, Ford JB, et al. Trends in postpartum hemorrhage in high resource countries: a review and recommendations from the International Postpartum Hemorrhage Collaborative Group. BMC pregnancy childbirth. 2009;9(1):55.

8. New JR, Cahill N, Stover J, Gupta YP, Alkema L. Levels and trends in contraceptive prevalence, unmet need, and demand for family planning for 29 states and union territories in India: a modelling study using the Family Planning Estimation Tool. Lancet Glob Health. 2017;5(3):e350-8.

9. Rosa CQ, Silveira DS, Costa JS. Factors associated with lack of prenatal care in a large municipality. Revista de saude publica. 2014;48:977-84.

10. Radhakrishnan T, Vasanthakumari KP, Babu PK. Increasing trend of caesarean rates in India: Evidence from NFHS-4. JMSCR. 2017;5(8):26167-76.

Cite this article as: Agrawal S, Singh A, Biswas R, Singh A. Retrospective review of maternal deaths and maternal near misses due to major obstetric haemorrhage at a tertiary care centre in India. Int $\mathbf{J}$ Reprod Contracept Obstet Gynecol 2019;8:3431-4. 